\title{
Mutated KRAS is an Independent Negative Prognostic Factor for Survival in NSCLC Stage III Disease Treated with High-Dose Radiotherapy
}

\author{
A. Hallqvist, ${ }^{1,2}$ F. Enlund, ${ }^{3}$ C. Andersson, ${ }^{3}$ H. Sjögren, ${ }^{4}$ \\ A. Hussein, ${ }^{3}$ E. Holmberg, ${ }^{1}$ and J. Nyman ${ }^{1}$ \\ ${ }^{1}$ Department of Oncology, Institute of Clinical Sciences, Sahlgrenska Academy at University of Gothenburg, \\ 40530 Gothenberg, Sweden \\ ${ }^{2}$ Department of Oncology, Sahlgrenska University Hospital, 41345 Gothenburg, Sweden \\ ${ }^{3}$ Department of Pathology, Sahlgrenska University Hospital, 41345 Gothenburg, Sweden \\ ${ }^{4}$ Department of Cytogenetics, Sahlgrenska University Hospital, 41345 Gothenburg, Sweden
}

Correspondence should be addressed to A. Hallqvist, andreas.hallqvist@oncology.gu.se

Received 8 May 2012; Revised 10 July 2012; Accepted 14 August 2012

Academic Editor: Elisabeth Quoix

Copyright ( 2012 A. Hallqvist et al. This is an open access article distributed under the Creative Commons Attribution License, which permits unrestricted use, distribution, and reproduction in any medium, provided the original work is properly cited.

Background. The main attention regarding prognostic and predictive markers in NSCLC directs towards the EGFR-targeted pathway, where the most studied genetic alterations include EGFR mutations, EGFR copy number, and KRAS mutations. We wanted to explore the prognostic impact of mutated KRAS in the stage III setting treated with high-dose radiochemotherapy. Methods. Samples were obtained from patients participating in two prospective studies of locally advanced NSCLC receiving combined radiochemotherapy: the RAKET study, a randomized phase II study where patients were treated with induction chemotherapy (carboplatin/paclitaxel) followed by concurrent radiochemotherapy, and the Satellite trial, a phase II study with induction chemotherapy (cisplatin/docetaxel) followed by radiotherapy concurrent cetuximab. The samples were analysed regarding KRAS mutations, EGFR mutations, and EGFR FISH positivity. Results. Patients with mutated KRAS had a significantly inferior survival, which maintained its significance in a multivariate analysis when other possible prognostic factors were taken into account. The prevalence of KRAS mutations, EGFR mutations, and EGFR FISH positivity were 28.8\%, 7.5\%, and 19.7\%, respectively. Conclusion. Mutated KRAS is an independent negative prognostic factor for survival in NSCLC stage III disease treated with combined radiochemotherapy. The prevalence of KRAS mutations and EGFR mutations are as expected in this Scandinavian population.

\section{Introduction}

The importance of finding prognostic and predictive markers is an ongoing challenge in oncology. The main attention regarding NSCLC directs towards the epidermal growth factor receptor- (EGFR) targeted pathway, where the most studied genetic alterations include EGFR mutations, EGFR copy number, and KRAS mutations. KRAS mutations have been shown to be associated with worse survival in resected patients $[1,2]$ and have also been shown to be a negative prognostic factor regarding adjuvant chemotherapy [3], whereas the significance in the stage III (locally advanced) or stage IV (metastatic) setting still is unclear.

In this study, we analysed tumour tissue samples regarding KRAS mutations, EGFR mutations, and EGFR positivity by FISH-high polysomy and amplification as defined by Cappuzzo et al. [4] - in patients with NSCLC stage III disease. The study population is represented by patients from two prospective trials who have received combined radiochemotherapy with curative intent: The RAKET study, a randomized three-armed phase II study where patients were treated with two cycles of induction chemotherapy 
(carboplatin/paclitaxel) followed by either hyperfractionated accelerated radiotherapy concurrent with a third cycle chemotherapy, conventional radiotherapy concurrent with daily paclitaxel or conventional radiotherapy concurrent with weekly paclitaxel [5]. Secondly the recently finished Satellite trial, a one-armed phase II study, where the treatment consisted of two cycles of induction chemotherapy (cisplatin/docetaxel) followed by radiotherapy concurrent with the EGFR-directed antibody cetuximab [6]. These two studies had the same inclusion criteria.

The aim was to explore the prognostic impact of KRAS mutations in the stage III setting where the patients have been treated with high-dose radiotherapy with concurrent chemotherapy/biotherapy and to survey the prevalence of KRAS and EGFR alterations in a Scandinavian population.

\section{Patients and Methods}

2.1. Patients. The patients involved in these analyses participated in the RAKET or Satellite study. The inclusion criteria were identical and consisted of histologically or cytologically proven nonresectable NSCLC stage IIIA/B (the 6th version of the TNM system), age $>18$, FEV1 $>1.0 \mathrm{~L}$ or $40 \%$, PS $0-1$, and adequate bone marrow reserve. Main exclusion criteria were malignant pleural effusion, other malignancy treated within the last five years. Neither study excluded patients with weight loss. The RAKET study is a randomized national multicentre three-armed phase II study where patients were treated with two cycles of induction chemotherapy (carboplatin AUC 6/paclitaxel $200 \mathrm{mg} / \mathrm{m}^{2}$ ) followed by either (a) hyperfractionated accelerated radiotherapy 1.7 Gy BID to $64.6 \mathrm{~Gy}$ concurrent with a third-cycle chemotherapy, (b) radiotherapy with $2 \mathrm{~Gy}$ daily to $60 \mathrm{~Gy}$ concurrent with daily paclitaxel $12 \mathrm{mg} / \mathrm{m}^{2}$ or (c) radiotherapy with 2 Gy daily to $60 \mathrm{~Gy}$ concurrent with weekly paclitaxel $60 \mathrm{mg} / \mathrm{m}^{2}$. The study included 151 patients between 2002 and 2005. The Satellite trial is a one-armed multicentre phase II study where patients were treated with two cycles of induction chemotherapy (cisplatin $75 \mathrm{mg} / \mathrm{m}^{2} /$ docetaxel $75 \mathrm{mg} / \mathrm{m}^{2}$ ) followed by radiotherapy, 2 Gy daily, to $68 \mathrm{~Gy}$ concurrent with weekly cetuximab (initial dose of $400 \mathrm{mg} / \mathrm{m}^{2}$ followed by $250 \mathrm{mg} / \mathrm{m}^{2}$ ). Seventy-five patients were recruited during 2006-2007. All patients gave their written informed consent and the studies were approved by the regional ethics board.

2.2. Tissue Specimen. Formalin-fixed paraffin-embedded tissues were collected from the diagnostic/pretherapeutic samples, and the analyses were performed at one site. The specimens were pathologically revised by a reference pathologist, and the section with highest percentage of tumour cells were estimated and marked, thereafter microdissected in preparation for the molecular analyses.

2.3. Molecular Analyses. The analysis of the KRAS- and EGFR mutations were performed by using TheraScreen KRAS Mutation Kit and TheraScreen EGFR29 Mutation Kit (DxS Diagnostics), detecting mutations in exon 2 and exon 18, 19, 21, respectively. EGFR copy number was analysed by using Vysis LSI EGFR SpectrumOrange/CEP7 SpectrumGreen Probe (Vysis Inc. Abbot laboratories), where the findings were classified according to Capuzzo et al. hence the term FISH positivity comprises high polysomy and amplification [4].

2.4. Statistics. The statistics for the main studies have been previously described $[5,6]$. The OS survival analyses are done according to the Kaplan Meier method and possible univariate differences between groups are estimated with logrank test. Univariate and multivariate Cox regression were done for several prognostic factors (i.e., KRAS mutation, performance status, gender, stage, and weight loss). In the multivariate Cox analyses the most significantly covariate was kept in the model and the other variables were entered into the model one by one and were maintained if they statistically improved the model on the $5 \%$ level.

\section{Results}

3.1. Main Results in the RAKET and Satellite Trials. The studies are as mentioned published previously but in short patients in the Satellite trial had a median survival of 17 months with a toxicity profile inferior to what usually is seen in concurrent chemoradiation. The RAKET trial had a median survival of 17.8 months, with all arms showing acceptable side effects, however there was no difference between the arms regarding local control or survival $[5,6]$.

3.2. Tissue Availability. All specimens from the patients in the Satellite trial were collected that previously had a biopsy performed, which were 34 out of 71 that is, $48 \%$, the remaining patients were diagnosed on cytology. A group of the same size was chosen from the RAKET study using all tissue samples from the institution that randomized most of the patients. We hereby obtained 35 samples and as the number equals the sample number in the Satellite study, we then made a comparison between baseline characteristics in the whole study population and the subset with tissue specimen available (Table 1). The groups with tissue samples available show close to the same basal criteria as the whole population, with the exception of a slightly higher proportion of adenocarcinoma in the RAKET study group.

The mean percentage of tumour cells in the samples, as assessed by the reference pathologist was 79\% (range 10$100 \%)$.

3.3. KRAS Mutational Status (Tables 2 and 3). The prevalence of KRAS mutations was $28.8 \%$ (19 out of 66 ), where the majority was found in adenocarcinomas and a small proportion in squamous cell carcinoma (SCC, 10.5\%). The group with mutated KRAS had a significantly inferior survival, which maintained its significance when introducing other possible prognostic factors into the cox regression model. They had previously shown to be of importance in the main study analyses, or are known to influence survival (i.e., performance status, gender, stage, and weight loss). The group difference is shown in Figure 1 with a hazard ratio of 
TABLE 1: Baseline characteristics in the whole study population and the subset with tissue specimen available.

\begin{tabular}{|c|c|c|c|c|}
\hline & \multicolumn{2}{|c|}{ RAKET } & \multicolumn{2}{|c|}{ Satellite } \\
\hline & Main study & Examined group & Main study & Examined group \\
\hline$n=$ & 151 & 35 & 71 & 34 \\
\hline Median age & $62(43-78)$ & $61(49-78)$ & $62(42-81)$ & $62(43-76)$ \\
\hline Gender F/M & $48 / 52 \%$ & $46 / 54 \%$ & $51 / 49 \%$ & $53 / 47 \%$ \\
\hline PS $0 / 1$ & $55 / 45 \%$ & $40 / 60 \%$ & $62 / 38 \%$ & $65 / 35 \%$ \\
\hline Stage IIIA/IIIB & $34 / 66 \%$ & $29 / 71 \%$ & $37 / 62 \%$ & $24 / 76 \%$ \\
\hline Weight loss $>5 \%$ & $17 \%$ & $26 \%$ & $37 \%$ & $32 \%$ \\
\hline FEV1 & $2.1(0.8-4.5)$ & $2.2(0.9-4.3)$ & $2.2(1.1-3.7)$ & $2.1(1.1-3.7)$ \\
\hline \multicolumn{5}{|l|}{ Histology: } \\
\hline Adenocarcinoma & $48 \%$ & $63 \%$ & $49 \%$ & $44 \%$ \\
\hline SCC & $32 \%$ & $26 \%$ & $39 \%$ & $44 \%$ \\
\hline NSCLC NOS & $20 \%$ & $11 \%$ & $12 \%$ & $12 \%$ \\
\hline
\end{tabular}

TABLe 2: Prevalence, and relation to patient characteristics of EGFR mutations, KRAS mutations, and EGFR FISH positivity.

\begin{tabular}{|c|c|c|c|}
\hline & EGFRmut & EGFR FISHpos & KRASmut \\
\hline$n=$ & $5=7.5 \%$ & $12=19.7 \%$ & $19=28.8 \%$ \\
\hline Inconclusive & 2 & 8 & 3 \\
\hline Median age & $62(47-71)$ & $62(43-75)$ & $62(55-73)$ \\
\hline Gender F/M & $60 / 40 \%$ & $67 / 33 \%$ & $58 / 42 \%$ \\
\hline PS $0 / 1$ & $40 / 60 \%$ & $50 / 50 \%$ & $48 / 52 \%$ \\
\hline Stage IIIA/IIIB & $40 / 60 \%$ & $17 / 83 \%$ & $21 / 79 \%$ \\
\hline Weight loss $>5 \%$ & $20 \%$ & $42 \%$ & $32 \%$ \\
\hline \multicolumn{4}{|l|}{ Histology } \\
\hline Adenocarcinoma & $40 \%$ & $42 \%$ & $68.5 \%$ \\
\hline SCC & $40 \%$ & $42 \%$ & $10.5 \%$ \\
\hline NSCLC NOS & $20 \%$ & $16 \%$ & $21 \%$ \\
\hline
\end{tabular}

$2.32(P=0.006,95 \%$ CI $1.27-4.26)$. The complete univariate and multivariate analyses are shown in Tables 4 and 5.

3.4. EGFR Mutational Status (Tables 2 and 3). The prevalence of EGFR mutations were $7.5 \%$ (5 out of 67 ), one exon 19 deletion, one L858R (exon 21) mutation both in adenocarcinomas and three G719X mutations (exon 18) which were found in squamous cell carcinomas (SCC) and NSCLC NOS.

3.5. EGFR Gene Copy Number (Tables 2 and 3). The prevalence of FISH positivity regarding EGFR was 19.7\% (12 out of 61), where the prevalence in the Satellite and RAKET trial differed substantially $32.3 \%$ and $6.7 \%$ respectively. The histologies involved were in equal amounts SCC and adenocarcinoma with a lesser proportion NSCLC NOS. Four of the patients that were FISH positive had overlapping mutations, two with mutated KRAS, and two with mutated EGFR.

\section{Discussion}

The knowledge about KRAS mutations and EGFR alterations, and their role in NSCLC, has expanded considerably

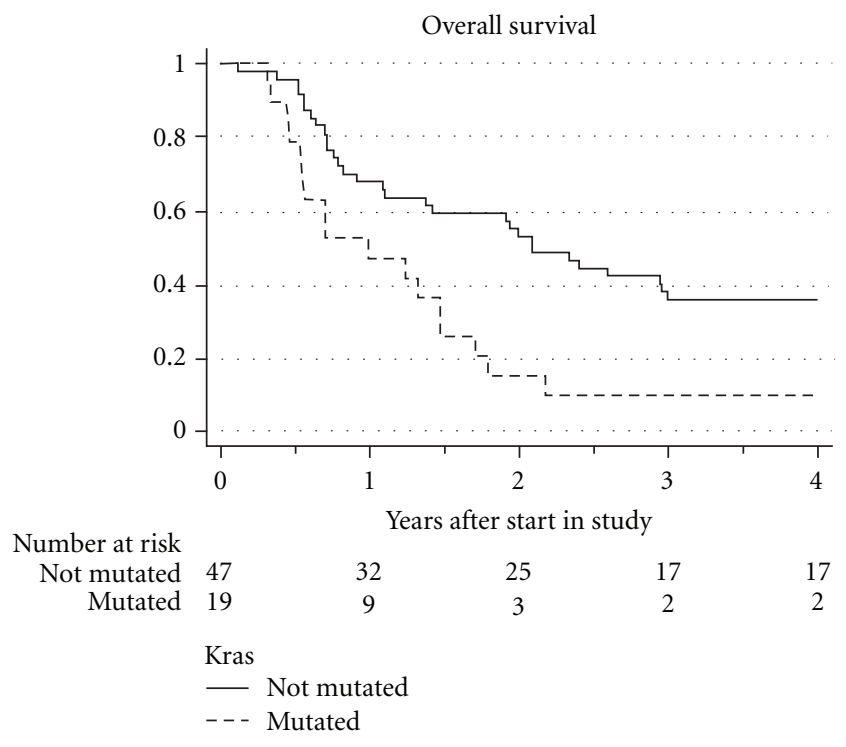

FIGURE 1: Survival depending on KRAS mutational status in the whole study population (HR 2.32, $P=0.006,95 \%$ CI $1.27-4.26$ ).

in the last decade. KRAS mutations are more common in adenocarcinomas and smokers and are reported in 15$30 \%$ without any pronounced ethnical differences [7-10]. KRAS mutations have been shown to be associated with worse survival or higher frequency of relapses in resected patients $[1,2]$. In a meta-analysis regarding all NSCLC stages, KRAS was a negative prognostic factor in terms of survival in univariate analysis but it was not analysed in the multivariate setting [11]. There is also data that KRAS is a negative prognostic factor for treatment with adjuvant chemotherapy $[3,8]$, whereas data regarding chemotherapy in the metastatic setting still is unclear. We have only found one study on the matter in stage III disease in which the patients received neoadjuvant radiochemotherapy followed by surgery where they found that KRAS was a negative prognostic factor regarding PFS in univariate analysis but a multivariate analysis was not performed [12]. In our study 
TABLE 3: Prevalence depending on trial subset (RAKET or Satellite).

\begin{tabular}{|c|c|c|c|c|c|c|}
\hline & \multicolumn{3}{|c|}{ RAKET } & \multicolumn{3}{|c|}{ Satellite } \\
\hline & EGFRmut & EGFR FISHpos & KRASmut & EGFRmut & EGFR FISHpos & KRASmut \\
\hline$n=$ & $2^{*}$ & $2^{* *}$ & 11 & $3^{\dagger}$ & $10^{\dagger \dagger}$ & 8 \\
\hline Inconclusive & 2 & 5 & 3 & & 3 & \\
\hline Median age & 69,71 & 69,75 & $60(55-73)$ & $47,54,62$ & $62(43-68)$ & $62(60-67)$ \\
\hline Gender F/M & F, F & $\mathrm{F}, \mathrm{M}$ & $55 / 45 \%$ & $\mathrm{~F}, \mathrm{M}, \mathrm{M}$ & $70 / 30 \%$ & $62 / 38 \%$ \\
\hline PS $0 / 1$ & 1,1 & 1,1 & $45 / 55 \%$ & $1,0,0$ & $60 / 40 \%$ & $50 / 50 \%$ \\
\hline Stage IIIA/IIIB & IIIB, IIIB & IIIB, IIIB & $18 / 82 \%$ & IIIA, 2IIIB & $20 / 80 \%$ & $25 / 75 \%$ \\
\hline Weight loss $>5 \%$ & 0 & 0,1 & $27 \%$ & $0,0,1$ & $40 \%$ & $37 \%$ \\
\hline \multicolumn{7}{|l|}{ Histology: } \\
\hline Adenocarcinoma & $100 \%$ & $100 \%$ & $64 \%$ & $0 \%$ & $30 \%$ & $75 \%$ \\
\hline SCC & $0 \%$ & $0 \%$ & $18 \%$ & $67 \%$ & $50 \%$ & $0 \%$ \\
\hline NSCLC NUS & $0 \%$ & $0 \%$ & $18 \%$ & $33 \%$ & $20 \%$ & $25 \%$ \\
\hline
\end{tabular}

*del 19 and L858R (exon 21).

** one overlapping with EGFRmut L858R.

${ }^{\dagger} \mathrm{G} 719 \mathrm{X}$ (exon 18).

${ }^{\dagger \dagger}$ two overlapping with KRASmut and one with G719X.

TABLe 4: Univariate Cox-analyses.

\begin{tabular}{|c|c|c|c|c|}
\hline Covariate & $\begin{array}{l}\text { Number of } \\
\text { patients }\end{array}$ & $\mathrm{HR}$ & $\begin{array}{l}\text { 95\% confidence } \\
\text { interval }\end{array}$ & $P$ value \\
\hline \multicolumn{5}{|l|}{ KRAS } \\
\hline Not mutated & 47 & 1 & & \\
\hline Mutated & 19 & 2.32 & $1.27-4.26$ & 0.006 \\
\hline \multicolumn{5}{|l|}{ Gender } \\
\hline Males & 38 & 1 & & \\
\hline Females & 31 & 0.68 & $0.38-1.21$ & 0.19 \\
\hline Age & 69 & 0.99 & $0.96-1.03$ & 0.67 \\
\hline \multicolumn{5}{|l|}{ PS } \\
\hline 0 & 36 & 1 & & \\
\hline 1 & 33 & 1.42 & $0.80-2.51$ & 0.23 \\
\hline \multicolumn{5}{|l|}{ Stage } \\
\hline IIIa & 18 & 1 & & \\
\hline IIIb & 49 & 1.99 & $0.96-4.14$ & 0.06 \\
\hline \multicolumn{5}{|l|}{ Weight loss } \\
\hline$<5 \%$ & 49 & & & \\
\hline$\geq 5 \%$ & 20 & 1.79 & $0.98-3.27$ & 0.06 \\
\hline \multicolumn{5}{|l|}{ Study } \\
\hline RAKET & 35 & 1 & & \\
\hline Satellite & 34 & 0.98 & $0.56-1.73$ & 0.95 \\
\hline
\end{tabular}

KRAS mutations were found in $28.8 \%$, predominantly in adenocarcinomas, and this is in accordance with published data. When analysing KRAS mutation as a prognostic marker we found mutated KRAS to be a significant negative prognostic marker for survival which kept its significance in multivariate analysis when introducing other known possible prognostic factors, that is, performance status, gender, stage and weight loss, into the model. As far as we know this is the first time this is shown in a multivariate analysis in stage III disease treated with radiochemotherapy with a curative intent. The treatment with cetuximab in the Satellite trial might have interfered, as mutated KRAS is a biomarker of resistance to cetuximab in colorectal cancer [13], however in neither the FLEX- nor BMS099 trial where cetuximab was given combined with chemotherapy in stage IV disease, a correlation between KRAS and response to cetuximab in NSCLC [9] was observed.

We have also presented the frequencies of EGFR mutations and EGFR FISH positivity in this cohort, solely to give an idea of the prevalence in this Scandinavian population. The frequency of EGFR mutations differ for example, depending on histology, smoking status, and ethnicity. The highest figures are seen in Asian female never smokers with adenocarcinomas ( $>50 \%)$, whereas Caucasians have an overall mutational frequency of $7-10 \%$ which will rise to about $13-16 \%$ when considering adenocarcinomas $[10,14-$ 16]. EGFR FISH positivity does not appear to have the same connection to histology or ethnicity but the prevalence varies substantially between 25 to $>50 \%$ [7, 9, 14, 15, 17-20].

In this study, the EGFR mutation prevalence of $7 \%$ in a histologically unselected northern European Caucasian population is in accordance with previously published data, but two out of five patients had tumors originating from SCC. The pattern seen in the present study, with several exon 18 mutations in SCC differing from the most frequently reported pattern with the most common mutations being exon 19 deletions and exon 21 mutations, predominantly in adenocarcinomas. This is probably due to small sample size. Regarding FISH positivity the prevalence seems to be somewhat low (19.7\%), with equal cases of adenocarcinomas and SCC. There is a substantial difference in the prevalence of FISH positive patients between the RAKET study and Satellite study where the prevalence is $6.7 \%$ and $32.3 \%$ respectively. We have no explanation for this difference, the samples were reanalysed with the same result. 
TABle 5: Multivariate Cox regression. In the multivariate Cox regression no other covariate than mutated KRAS significantly improved the model.

\begin{tabular}{|c|c|c|c|c|c|}
\hline Model number & Covariates & Number of patients & Hazard ratio (HR) & $P$ & 95\% confidence interval \\
\hline \multirow{6}{*}{ (1) } & Kras & & & & \\
\hline & Not mutated & 45 & 1.00 & & \\
\hline & Mutated & 19 & 2.32 & 0.010 & $1.21-4.13$ \\
\hline & Stage & & & & \\
\hline & IIIa & 16 & 1.00 & & \\
\hline & IIIb & 48 & 1.60 & 0.21 & $0.76-3.34$ \\
\hline \multirow{6}{*}{ (2) } & Kras & & & & \\
\hline & Not mutated & 47 & 1.00 & & \\
\hline & Mutated & 19 & 2.32 & 0.005 & $1.30-4.44$ \\
\hline & PS & & & & \\
\hline & 0 & 34 & 1.00 & & \\
\hline & 1 & 32 & 1.42 & 0.23 & $0.80-2.51$ \\
\hline \multirow{5}{*}{ (3) } & Kras & & & & \\
\hline & Not mutated & 47 & 1.00 & & \\
\hline & Mutated & 19 & 2.32 & 0.005 & $1.30-4.44$ \\
\hline & Weight loss & & & & \\
\hline & Continues (kg) & 66 & 1.05 & 0.30 & $0.95-1.15$ \\
\hline \multirow{6}{*}{ (4) } & Kras & & & & \\
\hline & Not mutated & 47 & 1.00 & & \\
\hline & Mutated & 19 & 2.28 & 0.008 & $1.24-4.20$ \\
\hline & Sex & & & & \\
\hline & Males & 38 & & & \\
\hline & Females & 31 & 0.63 & 0.132 & $0.35-1.15$ \\
\hline \multirow{5}{*}{ (5) } & Kras & & & & \\
\hline & Not mutated & 47 & 1.00 & & \\
\hline & Mutated & 19 & 2.28 & 0.007 & $1.27-4.27$ \\
\hline & Age & & & & \\
\hline & Continues (year) & 66 & 0.99 & 0.524 & $0.95-1.03$ \\
\hline \multirow{6}{*}{ (6) } & Kras & & & & \\
\hline & Not mutated & 47 & 1.00 & & \\
\hline & Mutated & 19 & 2.32 & 0.007 & $1.26-4.26$ \\
\hline & Study & & & & \\
\hline & RAKET & 32 & 1.00 & 0.77 & $0.52-1.63$ \\
\hline & Satellite & 34 & 0.92 & & \\
\hline
\end{tabular}

In summary, we have shown that mutated KRAS is an independent negative prognostic factor for survival in NSCLC stage III disease treated with combined chemoradiotherapy or bioradiotherapy, and the prevalence of KRAS mutations and EGFR mutations are as expected in this northern European population.

\section{Conflict of Interests}

The authors declare that there is no potential conflict of interest.

\section{Acknowledgment}

The authors would like to thank Merck Serono for an unrestricted grant making it possible to accomplish the study.

\section{References}

[1] Y. T. Kim, T. Y. Kim, D. S. Lee et al., "Molecular changes of epidermal growth factor receptor (EGFR) and KRAS and their impact on the clinical outcomes in surgically resected adenocarcinoma of the lung," Lung Cancer, vol. 59, no. 1, pp. 111-118, 2008.

[2] T. Woo, K. Okudela, T. Yazawa et al., "Prognostic value of KRAS mutations and Ki-67 expression in stage I lung adenocarcinomas," Lung Cancer, vol. 65, no. 3, pp. 355-362, 2009.

[3] M. S. Tsao, S. Aviel-Ronen, K. Ding et al., "Prognostic and predictive importance of p53 and RAS for adjuvant chemotherapy in non-small-cell lung cancer," Journal of Clinical Oncology, vol. 25, no. 33, pp. 5240-5247, 2007.

[4] F. Cappuzzo, F. R. Hirsch, E. Rossi et al., "Epidermal growth factor receptor gene and protein and gefitinib sensitivity in non-small-cell lung cancer," Journal of the National Cancer Institute, vol. 97, no. 9, pp. 643-655, 2005. 
[5] J. Nyman, S. Friesland, A. Hallqvist et al., "How to improve loco-regional control in stages IIIa-b NSCLC? Results of a three-armed randomized trial from the Swedish lung cancer study group," Lung Cancer, vol. 65, no. 1, pp. 62-67, 2009.

[6] A. Hallqvist, G. Wagenius, H. Rylander et al., "Concurrent cetuximab and radiotherapy after docetaxel-cisplatin induction chemotherapy in stage III NSCLC: satellite-a phase II study from the Swedish lung cancer study group," Lung Cancer, vol. 71, no. 2, pp. 166-172, 2011.

[7] X. Zhang and A. Chang, "Molecular predictors of EGFRTKI sensitivity in advanced non-small cell lung cancer," International Journal of Medical Sciences, vol. 5, no. 4, pp. 209217, 2008.

[8] G. J. Riely, J. Marks, and W. Pao, "KRAS mutations in nonsmall cell lung cancer," Proceedings of the American Thoracic Society, vol. 6, no. 2, pp. 201-205, 2009.

[9] S. Khambata-Ford, C. T. Harbison, L. L. Hart et al., "Analysis of potential predictive markers of cetuximab benefit in BMS099, a phase III study of cetuximab and first-line taxane/carboplatin in advanced non-small-cell lung cancer," Journal of Clinical Oncology, vol. 28, no. 6, pp. 918-927, 2010.

[10] W. Zhou and D. C. Christiani, "East meets West: ethnic differences in epidemiology and clinical behaviors of lung cancer between East Asians and Caucasians," Chinese Journal of Cancer, vol. 30, no. 5, pp. 287-292, 2011.

[11] C. Mascaux, N. Iannino, B. Martin et al., "The role of RAS oncogene in survival of patients with lung cancer: a systematic review of the literature with meta-analysis," British Journal of Cancer, vol. 92, no. 1, pp. 131-139, 2005.

[12] P. Broermann, K. Junker, B. H. Brandt et al., "Trimodality treatment in stage III nonsmall cell lung carcinoma: prognostic impact of K-ras mutations after neoadjuvant therapy," Cancer, vol. 94, no. 7, pp. 2055-2062, 2002.

[13] M. W. Saif and M. Shah, "K-Ras mutations in colorectal cancer: a practice changing discovery," Clinical Advances in Hematology and Oncology, vol. 7, no. 1, pp. 45-64, 2009.

[14] C. Q. Zhu, G. da Cunha Santos, K. Ding et al., "Role of KRAS and EGFR as biomarkers of response to erlotinib in National Cancer Institute of Canada clinical trials group study BR.21," Journal of Clinical Oncology, vol. 26, no. 26, pp. 4268-4275, 2008.

[15] R. Morinaga, I. Okamoto, Y. Fujita et al., "Association of epidermal growth factor receptor (EGFR) gene mutations with EGFR amplification in advanced non-small cell lung cancer," Cancer Science, vol. 99, no. 12, pp. 2455-2460, 2008.

[16] A. Rossi, D. Galetta, and C. Gridelli, "Biological prognostic and predictive factors in lung cancer," Oncology, vol. 77, no. 1, pp. 90-96, 2010.

[17] T. Sone, K. Kasahara, H. Kimura et al., "Comparative analysis of epidermal growth factor receptor mutations and gene amplification as predictors of gefitinib efficacy in Japanese patients with nonsmall cell lung cancer," Cancer, vol. 109, no. 9, pp. 1836-1844, 2007.

[18] T. J. Pugh, G. Bebb, L. Barclay et al., "Correlations of EGFR mutations and increases in EGFR and HER2 copy number to gefitinib response in a retrospective analysis of lung cancer patients," BMC Cancer, vol. 7, article 128, 2007.

[19] F. R. Hirsch, R. S. Herbst, C. Olsen et al., "Increased EGFR gene copy number detected by fluorescent in situ hybridization predicts outcome in non-small-cell lung cancer patients treated with cetuximab and chemotherapy," Journal of Clinical Oncology, vol. 26, no. 20, pp. 3351-3357, 2008.

[20] F. R. Hirsch, M. Varella-Garcia, P. A. Bunn Jr. et al., "Epidermal growth factor receptor in non-small-cell lung carcinomas: correlation between gene copy number and protein expression and impact on prognosis," Journal of Clinical Oncology, vol. 21, no. 20, pp. 3798-3807, 2003. 


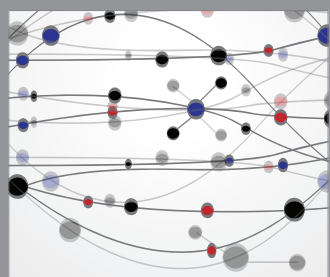

The Scientific World Journal
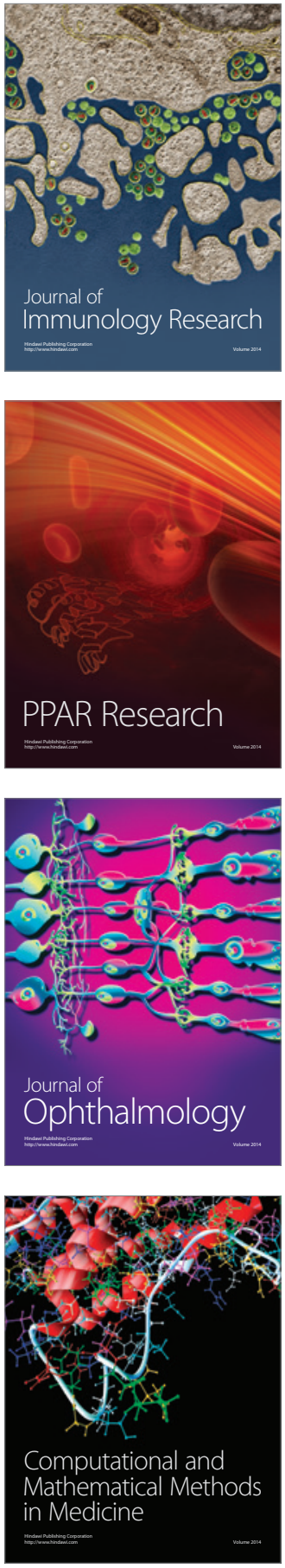

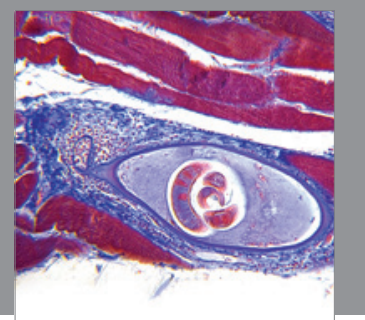

Gastroenterology

Research and Practice
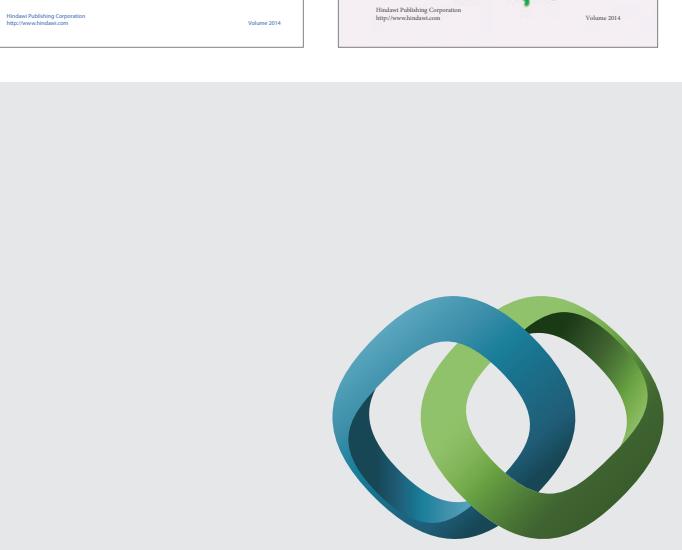

\section{Hindawi}

Submit your manuscripts at

http://www.hindawi.com
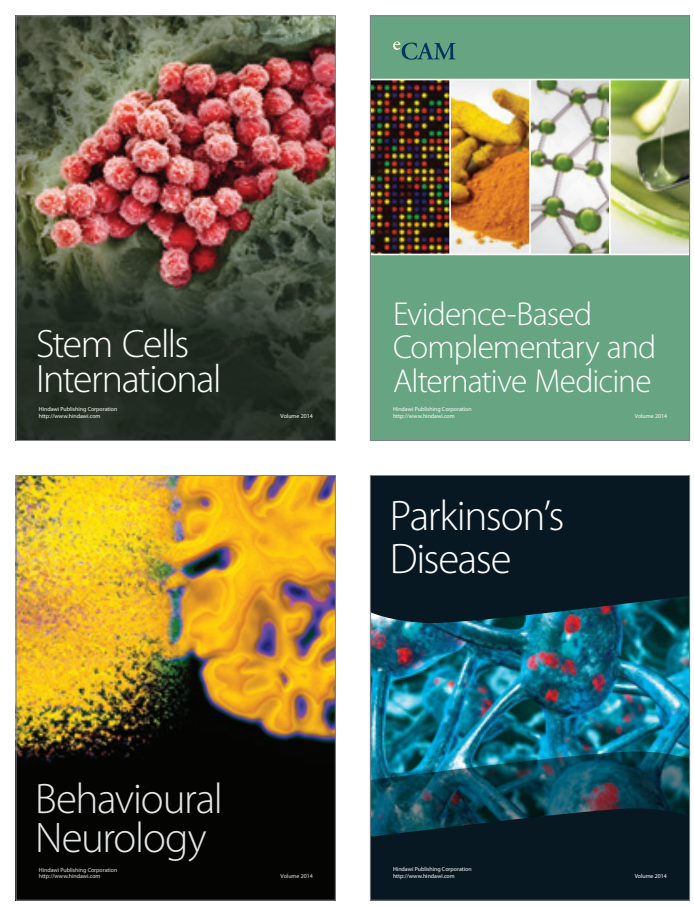

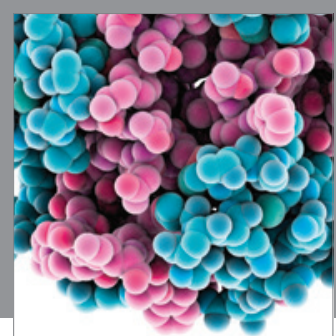

Journal of
Diabetes Research

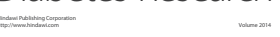

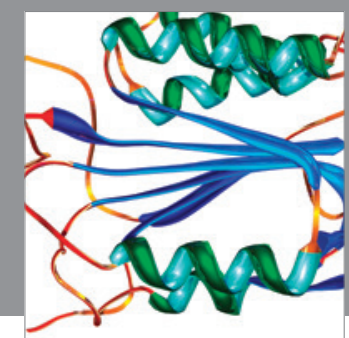

Disease Markers
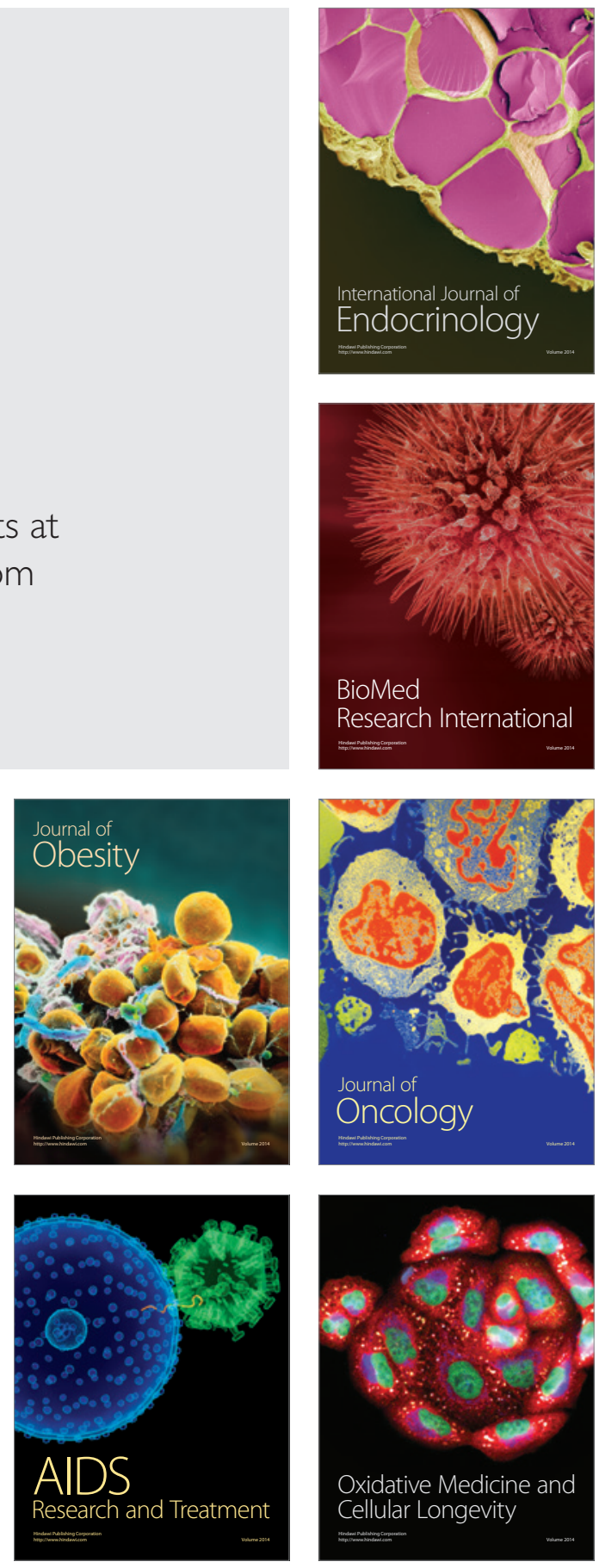